\title{
Synthesis of 7-Azaserotonin: Its Photophysical Properties Associated with Excited State Proton Transfer Reaction
}

\author{
Pei-Wen Wu, ${ }^{\dagger}$ Wan-Ting Hsieh, ${ }^{\dagger}{ }^{\top}$ Yi-Ming Cheng, ${ }^{\dagger}$ Ching-Yen Wei, ${ }^{\ddagger}$ and Pi-Tai Chou ${ }^{*}, \dagger$ \\ Department of Chemistry, National Taiwan University, Taipei 106, Taiwan, and Industrial Technology Research \\ Institute, Chemical Engineering Division, Room 313, Building 17, 321, Kuang Fu Road, Section 2,
} Hsin Chu 300, Taiwan

Received June 19, 2006; E-mail: chop@ntu.edu.tw

\section{Introduction}

Perhaps one of the most prominent neurotransmitters is serotonin (3-(2-aminoethyl)-1H-indol-5-ol, 1a in Scheme 1), which is a completely natural hormone ${ }^{1}$ and has a powerful effect on most parts of the brain involved in controlling vital functions such as behavior, movement, pain, sexual activity, and appetite. In many neuropsychiatric disorders, ${ }^{2}$ the serotonin system may also play a key modulatory role. Although the pathophysiology of many neuropsychiatric disorders remains enigmatic, abnormalities in serotonin signaling have been strongly implicated.

That such a small molecule like serotonin acts as a vital element in brain function has attracted much attention in synthetic chemistry. Focus on derivatization of serotonin has been one of the intensive areas in medicinal chemistry. ${ }^{3}$ While most of the approaches lie in the substitution effect on the parent serotonin, we are interested in replacing the parent indole moiety with the 7-azaindole chromophore, forming 7-azaserotonin (3-(2-aminoethyl)-5-ol-1H-pyrrolo$[2,3-b]$ pyridine, 1 in Scheme 1). Studies have shown great differences in both the physical and photophysical properties between indole and azaindole systems. It has been well-established that HOMO and LUMO for indole (azaindole) are ascribed to pyrrole and benzene (pyridine) rings, respectively. ${ }^{4}$ In comparison to indole, the electron withdrawing properties of pyridyl nitrogen in azaindole should lead to a further decrease of the LUMO level, resulting in a smaller energy gap. Furthermore, the lower LUMO level prevents the electron ejection upon electronic excitation. As a prototypical example, 7-azatryptophan has replaced tryptophan in numerous biological applications ${ }^{5}$ owing to its longer wavelength absorption $(>300 \mathrm{~nm})$, which can be free of the interference by natural tryptophan or other biochromophores. Moreover, its singleexponential decay dynamics makes it apparently superior to tryptophan, which normally gives rise to complications due to its biexponential decay dynamics. ${ }^{6}$

In addition to the above advantages, in view of its biochemical properties, 1 may possess an additional pyridyl nitrogen capable of hydrogen-bonding that may be more affinitive to the receptor of 1a, such that $\mathbf{1}$ may act as a potential agonist or antagonist of 1a receptors. Herein, we report the first synthesis of $\mathbf{1}$ and its intriguing photophysical properties, namely the excited-state protontransfer reaction.

The 7-step synthetic route to $\mathbf{1}$ is depicted in Scheme 1, in which commercially available 7-azaindole served as a starting material. Step 1 involved halogenation with bromine in a mixture of $t$-butanol and water at room temperature to provide the tribromo derivative $2(85 \%) .{ }^{7}$ In step 2, 2 was then treated with zinc in acetic acid to furnish 3 in $95 \%$ yield. Reduction of the amide function was realized with the borane-tetrahydrofuran complex, and the resulting indoline

$\dagger$ National Taiwan University

¥ Industrial Technology Research Institute.
Scheme 1. (a) Synthesis of 7-Azaserotonin and (b) the Structure of Serotonin

(a)
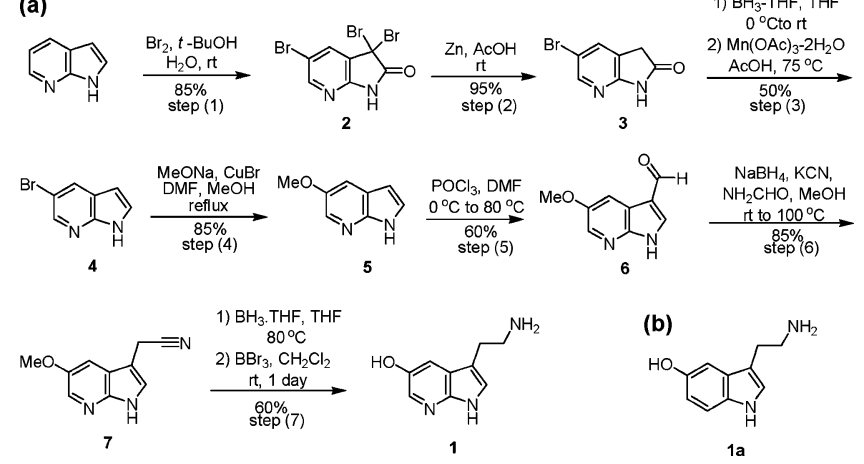

was oxidized with manganese triacetate in acetic acid at $75{ }^{\circ} \mathrm{C}$ to give 4 with $50 \%$ yield. Subsequently, the 5-methoxy functionalization was established by step 4 , in which compound $\mathbf{4}$ was treated in a mixture of $N, N$-dimethylformamide and methanol with sodium methoxide in the presence of copper(I) bromide, yielding $\mathbf{5}$ in $85 \%$ yield. In step 5, to elaborate the derivatization of the 3-position of 7 -azaindole moiety, $\mathbf{5}$ was subjected to the Vilsmeier' reaction, and 6 was achieved successfully in $60 \%$ yield.

In step 6, 6 was first treated with sodium borohydride in binary solvents and then reacted with $\mathrm{KCN}$. Note that this step is strongly solvent-mixture dependent. Among various mixtures, $\mathrm{MeOH} / \mathrm{NH}_{2}-$ CHO $(1: 1, v / v)$ maximized the yield of 7 to $85 \%$.

Subsequently, reduction of the cyano group to the corresponding amine was performed. The first attempt, incorporating reduction under hydrogen gas by Raney Ni catalyst, was not efficient. Alternatively, reduction of the cyano group was realized with a borane-THF complex. However, further purification of the resulting amine derivative was subject to decomposition. To avoid this, without prepurification, we carried out the next step of the treatment with $\mathrm{BBr}_{3}$ and successfully transformed the methoxy group to the hydroxyl group, forming the target compound 7-azaserotonin (1). 1 was then purified by twice recrystallization from methanol. In summary, via Scheme 1, 1 has been successfully prepared with an overall yield of $\sim 10 \%$. Detailed synthetic procedures as well as characterization of the intermediates are elaborated in Supporting Information.

Figure 1 depicts the absorption and emission spectra of 1a and 1 in ethanol. In comparison, the first $S_{0}-S_{1}$ absorption peak of $\sim 320 \mathrm{~nm}$ is apparently red-shifted by $\sim 15 \mathrm{~nm}$ with respect to that of serotonin, supporting the lowering of LUMO and hence the decrease of the HOMO-LUMO energy gap by replacing the fused benzene ring (in 1a) with pyridine (in 1). Upon excitation, 1a exhibits a normal emission band with peak wavelength at $333 \mathrm{~nm}$ in ethanol, while in sharp contrast, dual emission appeared in $\mathbf{1}$, 


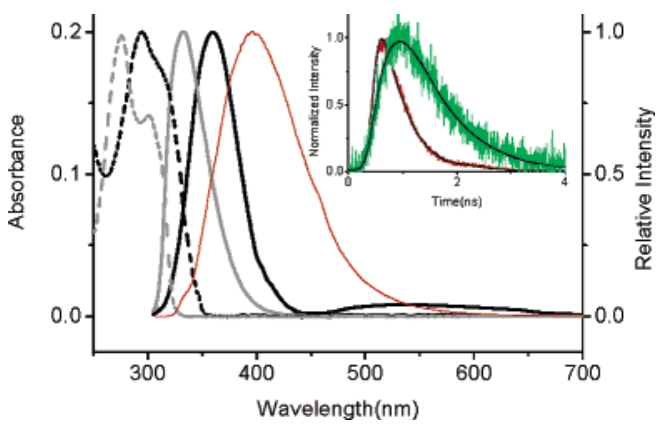

Figure 1. Absorption (dash) and emission (solid) spectra of 1a (gray) and $\mathbf{1}$ (black) in ethanol. Emission spectrum of $\mathbf{1}$ in $\mathrm{pH}=7$ is shown by the red solid line. The excitation wavelength is $300 \mathrm{~nm}$. Inset shows the relaxation dynamics of 1 in ethanol, monitored at $340 \mathrm{~nm}$ (red) and $540 \mathrm{~nm}$ (green).
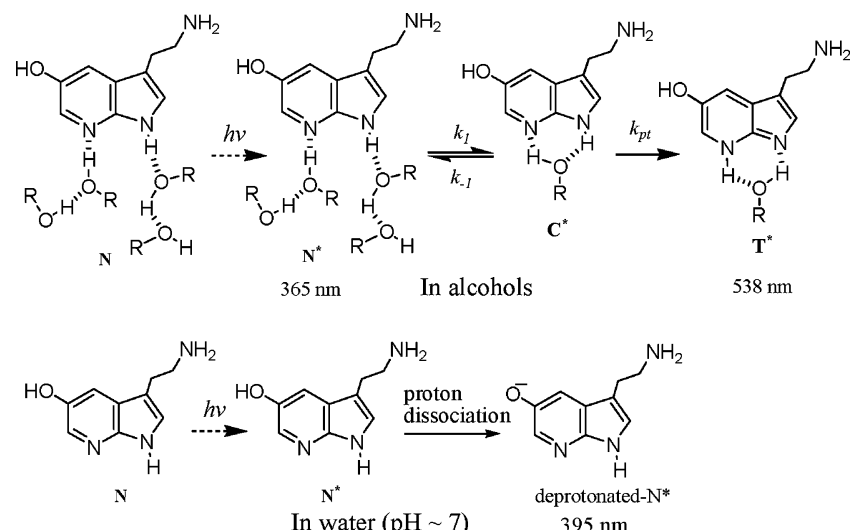

Figure 2. The proposed excited-state behavior of $\mathbf{1}$ in alcohol and water.

consisting of short wavelength (the $\mathrm{F}_{1}$ band) and long wavelength (the $F_{2}$ band) bands maximized at 365 (the $F_{1}$ band) and $538 \mathrm{~nm}$ (the $F_{2}$ band), respectively. The fluorescence excitation spectra monitored at the $F_{1}$ and $F_{2}$ bands are identical and are also the same as the absorption profile. The result eliminates the possibility that the dual emission is resulting from trace impurities. Furthermore, the ratio of the $F_{2}$ versus the $F_{1}$ band is concentration independent from $10^{-5}-10^{-3} \mathrm{M}$ in ethanol, discarding a proposal that the $F_{2}$ band originates from aggregation. Alternatively, the results can more plausibly be rationalized by the ethanol (or methanol) catalyzed proton-transfer reaction for $\mathbf{1}$ depicted in Figure 2. Figure 2 depicts a two-step proton-transfer mechanism, in which there exists a fast equilibrium between the 1:1 alcohol/1 cyclic HB structure $\left(\mathrm{C}^{*}\right)$ and other complexes generally described as 2:1 alcohol/1 noncyclic HB structure $\left(\mathrm{N}^{*}\right)$. Proton-transfer thus takes place from $\mathrm{C}^{*}$, resulting in a green proton-transfer tautomer $\left(\mathrm{T}^{*}\right)$ emission.

The scheme proposed above is based on a well-established mechanism associated with 7-azaindole and its corresponding analogues. ${ }^{8}$ Further support of this precursor-successor type of proton-transfer mechanism is rendered by the relaxation dynamics, in which a single-exponential decay time of $360 \pm 30 \mathrm{ps}$ for the $\mathrm{F}_{1}$ band (monitored at $340 \mathrm{~nm}$ ), within experimental error, correlates well with the $385 \pm 40 \mathrm{ps}$ risetime of the $\mathrm{F}_{2}$ band (monitored at $540 \mathrm{~nm}$, see the inset in Figure 1). Interestingly, however, a unique emission maximized at $395 \mathrm{~nm}\left(\tau_{\mathrm{f}} \approx 0.5 \mathrm{~ns}\right)$ was observed for $\mathbf{1}$ in aqueous solution $(\mathrm{pH} \approx 7$, see Figure 1$)$. The fact that we could not resolve any steady-state emission and rise-decay dynamics of the $\mathrm{F}_{2}$ band, in combination with the good fluorescence yield (0.15), leads us to propose that $\mathrm{H}_{2} \mathrm{O}$ assisted excited-state proton transfer may be prohibited in aqueous solution. We further carried out the luminescence study at $\mathrm{pH}=11.5$, in which the deprotonation of
$-\mathrm{OH}\left(\mathrm{p} K_{\mathrm{a}} \approx 9.6\right)$ should be $>95 \%$ (see Figure $\mathrm{S} 1$ in Supporting Information), and found that the corresponding emission spectrum $\left(\lambda_{\max }=395 \mathrm{~nm}\right.$, see Figure S1) is the same of that in $\mathrm{pH}=7$. In view of the different absorption spectra with the same emission profiles, we tentatively propose that the excited-state proton dissociation takes place in $\mathrm{pH}=7$, giving rise to a $395 \mathrm{~nm}$ anionic 1 emission. Given the evidence of large a Stokes-shifted $395 \mathrm{~nm}$ emission for $\mathbf{1}$ in neutral water, it is reasonable to expect that such an anionic species can be further stabilized by solvation, such that the further proton transfer is highly thermally unfavorable (see Figure 2). Studies in low $\mathrm{pH}(<5.0)$ are rather complicated owing to the protonation at the pyridyl nitrogen site.

In conclusion, the first synthesis and spectroscopic studies of $\mathbf{1}$ are reported. The differentiation of photophysics between alcohol (solvent catalyzed proton-transfer reaction) and water (deprotonation) are intriguing. In view of bioapplications, we thus suspect that once $\mathbf{1}$ is in certain hydrophilic medium, similar to that in alcohol, a water catalyzed excited-state proton-transfer reaction may take place, allowing it to serve as a suitable molecular probe. We also performed the molecular dynamic approaches for $\mathbf{1}$ using a homology model of human $5-\mathrm{HT}_{1 \mathrm{~B}}$ serotonin receptor to investigate the difference of interaction to the $5-\mathrm{HT}_{1 \mathrm{~B}}$ receptor between $\mathbf{1 a}$ and $\mathbf{1}$. Our preliminary data indicate that $\mathbf{1}$ has larger association strength than that of 1a, exhibiting its great potential to serve as a 5 - $\mathrm{HT}_{1 \mathrm{~B}}$ agonist or antagonist for the treatment of aggression or depression. We thus believe that exploration of $\mathbf{1}$ may spark a broad spectrum of interest in the fields of medicinal chemistry and biophysics.

Acknowledgment. This work is supported by the National Science Council and National Center for High-Performance Computing, Taiwan.

Supporting Information Available: Listing of experimental details and characterization data for all compounds. This material is available free of charge via the Internet at http://pubs.acs.org.

\section{References}

(1) (a) Barnes, N. M.; Sharp, T. Neuropharmacology 1999, 38, 1038. (b) Svenningsson, P.; Chergui, K.; Rachleff, I.; Flajolet, M.; Zhang, X.; Yacoubi, M. E.; Vaugeois, J. M.; Nomikos, G. G.; Greengard, P. Science 2006, 311, 77 .

(2) (a) Gingrich, J. A.; Hen, R. Psychopharmacology (Berlin), 2001, 155, 1. (b) Manji, H. K.; Drevets, W. C.; Charney, D. S. Nat. Med. 2001, 7, 541.

(3) (a) Flaugh, M. E.; Mullen, D. L.; Fuller, R. W.; Mason, N. R. J. Med. Chem. 1988, 31, 1746. (b) Malleron, J. L.; Gueremy, C.; Mignani, S.; Peyronel, J. F.; Truchon, A.; Blanchard, J. C.; Doble, A.; Laduron, P.; Piot, O. J. Med. Chem. 1993, 36, 1194. (c) Mattson, R. J.; Catt, J. D.; Denhart, D. J.; Deskus, J. A.; Ditta, J. L.; Higgins, M. A.; Marcin, L. R. Sloan, C. P.; Beno, B. R.; Gao, Q.; Cunningham, M. A.; Mattson, G. K.; Molski, T. F.; Taber, M. T.; Lodge, N. J. J. Med. Chem. 2005, 48, 6023.

(4) (a) Brause, R.; Krügler, D.; Schmitt, M.; Kleinermanns, K. J. Chem. Phys. 2005, 123, 224311. (b) Rogers, D. M.; Besley, N. A.; Hirst, J. D. J. Phys. Chem. B 2005, 109, 23061. (c) Kang, C.; Yi, J. T.; Pratt, D. W. Chem. Phys. Lett. 2006, 423, 7.

(5) (a) Chen, Y.; Gai, F.; Petrich, J. W. J. Phys. Chem. 1994, 98, 2203. (b) Rich, R. L.; Gai, F.; Lane, J. W.; Petrich, J. W.; Schwabacher, A. W. J. Am. Chem. Soc. 1995, 117, 733. (c) Kelepouris, L.; Blanchard, G. J. J. Phys. Chem. B 2002, 106, 6600.

(6) Chen, Y.; Rich, R. L.; Gai, F.; Petrich, J. W. J. Phys. Chem. 1993, 97, 1770 .

(7) Mazéas, D.; Guillaumet, G.; Viaud, M. C. Heterocycles 1999, 50, 1065

(8) (a) Chapman, C. F.; Maroncelli, M. J. Phys. Chem. 1992, 96, 8430. (b) Stratt, R. M.; Maroncelli, M. J. Phys. Chem. 1996, 100, 12981. (c) Chou, P. T.; Yu, W. S.; Wei, C. Y.; Cheng, Y. M.; Yang, C. Y. J. Am. Chem Soc. 2001, 123, 3599. (d) Chou, P. T.; Wu, G. R.; Wei, C. Y.; Shiao, M Y.; Liu, Y. I. J. Phys. Chem. A 2000, 104, 8863. (e) Chou, P. T.; Liao, J. H.; Wei, C. Y.; Yang, C. Y; Yu, W. S.; Chou, Y. H. J. Am. Chem. Soc. 2000, 122, 986. (f) Hung, F. T.; Hu, W. P.; Chou, P. T. J. Phys. Chem. A 2001, 105, 10475. (g) Yu, W. S.; Cheng, C. C.; Chang, C. P.; Wu, G. R.; Hsu, C. H.; Chou, P. T. J. Phys. Chem. A 2002, 106, 8006. (h) Chou, P. T.; Cheng, Y. M.; Yu, W. S.; Pu, S. C. J. Phys. Chem. A 2003, 107 5640. (i) Hu, W. P.; You, R. M.; Yen, S. Y.; Hung, F. T.; Chou, P. H Chou, P. T. Chem. Phys. Lett. 2003, 370, 139.

JA064310E 